\title{
Recommendations of using at least two different methods for measuring NO
}

\author{
Kapuganti J. Gupta ${ }^{1 *}$ and Abir U. Igamberdiev ${ }^{2 *}$ \\ ${ }^{1}$ Department of Plant Sciences, University of Oxford, Oxford, UK \\ 2 Department of Biology, Memorial University of Newfoundland, St. John's, NL, Canada \\ *Correspondence: jagadis.kapuganti@plants.ox.ac.uk; igamberdiev@mun.ca \\ Edited by: \\ Emmanuel Baudouin, Université Pierre et Marie Curie - Paris 6, France \\ Reviewed by: \\ Emmanuel Baudouin, Université Pierre et Marie Curie - Paris 6, France
}

\section{INTRODUCTION}

Nitric oxide (NO) is widely recognized as a signal molecule in plants. Various sources of NO were identified in plants (Moreau et al., 2010; Gupta et al., 2011; Mur et al., 2013) located in different compartments activated under various conditions. Briefly these are the mitochondrial nitrite: NO reductase reaction, the cytosolic nitrate reductase (NR), the plasma membrane nitrite: NO reductase (PM-NiNOR), xanthine oxidoreductase, NO synthase-like enzyme (putative), polyamine (PA)- and hydroxylamine (HA)-mediated pathways. $\mathrm{NO}$ acts as an intracellular messenger due to its diffusible capacity through various cellular compartments. After pioneering discovery of NO production in plants, scientists started digging deeply to the key function of NO and this research led to understanding of various roles of $\mathrm{NO}$ that include regulation of stomatal movement, root development, floral transition, response to biotic and abiotic stresses, symbiotic interactions. Despite of extensive research on $\mathrm{NO}$ roles in metabolism and signal transduction, its measurement remains challenging (Vandelle and Delledonne, 2008; Mur et al., 2011). We discuss below the problems associated with $\mathrm{NO}$ measurement and suggest some important solutions to tackle these problems.

The half-life of NO depends on its concentration and usually falls is in the range of ten(s) seconds. For instance, at $10 \mu \mathrm{M}$ concentration NO has a half-life of about $80 \mathrm{~s}$ whereas at $100 \mu \mathrm{M}$ concentration NO has a half-life of about $8 \mathrm{~s}$ (Wink and Mitchell, 1998). This means that at low concentration NO can easily diffuse from its origin to the site of action. At higher concentrations most of the NO rapidly undergoes autoxidation. Moreover NO half-life also depends on the presence of scavengers such as nonsymbiotic hemoglobin (class 1) and other major NO scavenging targets such as lipids and metal-containing proteins. Therefore, it is often very important to measure NO concentration which is crucial for understanding its function. But NO concentrations measured in same biological material by different methods often give different values (Mur et al., 2006; Planchet and Kaiser, 2006). In some cases NO production is localized to specific cells such as guard cells; therefore fluorescent probes are required to visualize NO producing sites. Here we describe why we need to use at least two different methods for measuring generation of $\mathrm{NO}$ in plants and suggest the best combination of methods for specific studies.

\section{OVERVIEW OF NO DETECTION METHODS: ADVANTAGES AND TECHNICAL PROBLEMS}

\section{MEASUREMENT OF NO IN THE GAS PHASE}

Chemiluminescence is a well-established method for $\mathrm{NO}$ measurement. In this method the reaction between $\mathrm{NO}$ and ozone $\left(\mathrm{O}_{3}\right)$ generates nitrogen dioxide $\left(\mathrm{NO}_{2}^{*}\right)$ in the excited-state which then emits a photon and reaches its ground state, a photomultiplier counts the light generated in the amount proportional to NO content. Chemiluminescence has been used to measure $\mathrm{NO}$ emissions from leaves, roots and from isolated mitochondria (Planchet et al., 2005; Gupta et al., 2011; Shah et al., 2013). This method is highly sensitive and can detect $\mathrm{NO}$ in the range of parts per billion which corresponds to picomolar concentrations in the tissue. The major disadvantage of this method is that it measures only the emitted NO in the gas phase (Planchet and Kaiser, 2006). Another disadvantage is that it measures only pure NO emitted from biological samples, while only a small portion (in green leaves of Arabidopsis less than $6 \%$ ) of the produced $\mathrm{NO}$ is emitted from the biological samples, the major part is quenched in the reaction with superoxide (Vanin et al., 2004), and in the hypoxic tissues scavenged by the nonsymbiotic hemoglobin (Igamberdiev et al., 2006).

Laser-based photoacoustic detection of NO uses the absorption of rapidly chopped infrared light by NO (Mur et al., 2011). The sound is generated during the absorption and relaxation which is detected by the microphone located in the photoacoustic cell. This method was used by Mur et al. (2005) to detect NO from tobacco leaves infected with bacteria (Pseudomonas). The advantage of this method is it very high precision, while the disadvantage is that $\mathrm{NO}$ can be detectable only in the gas phase and no special information about NO production in specific cells can be obtained. This method should be used together with DAF fluorescence (see below) to get the information about the presence of $\mathrm{NO}$ in the gas phase and in specific cells respectively.

Quantum cascade laser-based spectroscopic detection of $\mathrm{NO}$ (Moeskops et al., 2006; Gupta et al., 2013) represents another version of the previous method. The laser is integrated with a thermo-electrically cooled infrared detector. The detection sensitivity of this method is 0.03 parts per billion which is higher by $1-2$ orders of magnitude than in the photoacoustic detection. 
The Membrane Inlet Mass Spectrometry (MIMS) is a robust method for NO detection (Conrath et al., 2004). In this method the online detection of NO in gaseous phase is possible. The membrane barrier separates the sample from mass spectrometer and allows NO to be detected. Another advantage of MIMS is that, by using radioactive substrates, it is possible to detect the contribution of NO from each pathway by using radiolabelled arginine or nitrate/nitrite (Conrath et al., 2004). Though it is an excellent and very sensitive method, it was rarely used in plant NO research. This is probably due to its high cost and expertise requirement. In this method, as in other gas phase methods, the detection of oxidized forms of NO is not possible. But after measuring NO by this method, the samples can be ground and further analyzed by using mass spectrometer, indirect chemiluminescence, Griess reagent or NO electrodes.

\section{MEASUREMENT OF NO IN THE LIQUID PHASE}

A common method of measuring $\mathrm{NO}$ in liquid phase is based on using the NO electrodes. These include platinum/teflon or platinum/iridium (Pt/Ir) coated working electrode and $\mathrm{Ag} / \mathrm{AgCl}$ reference electrode. $\mathrm{NO}$ is detected via its oxidation at +0.8 to $+0.9 \mathrm{~V}$ compared to the reference electrode (Shibuki, 1990). NO electrodes have been used in plant NO research, e.g., to measure NO production in tobacco cells in response to cryptogein (Besson-Bard et al., 2008), to detect NO in fruits (Leshem, 1996), to measure NADH-dependent NO scavenging activity in plant extracts and in purified fractions containing class 1 hemoglobin (Igamberdiev et al., 2004, 2006). The disadvantage of this method is that it measures NO in the liquid phase and if plant tissues emit $\mathrm{NO}$ in the gaseous phase, it is not quite reliable to detect it using these electrodes. The best combination is the use of the chemiluminescence method to detect NO in the gas phase and then NO electrodes to check NO concentration in the extracts of plant tissues.

As we have mentioned above, NO is highly reactive and only its small portion is emitted from the biological samples while the rest is oxidized (Vanin et al., 2004), therefore it is not possible to measure oxidized forms of NO such as nitrate, nitrite by gas phase chemiluminescence. But this limitation can be overcome by using indirect chemiluminescence in which nitrate and nitrite produced from oxidation of $\mathrm{NO}$ are reduced back to NO by injecting sample extracts into boiling acidic vanadium chloride (Gupta and Kaiser, 2010).

If there is no proper equipment for doing indirect chemiluminescence, then the alternative method to measure oxidized forms of NO is the Griess reagent assay, which is relatively cheap. In this method NO is oxidized to nitrite which reacts with sulphanilic acid and $\alpha$ naphthylamine under acidic conditions to produce the azodye which can be detected at $520 \mathrm{~nm}$. This method is not commonly used by plant scientists but there are few reports (Shirinova et al., 1993; Planchet et al., 2005). Sensitivity is very low for this method $(0.5 \mu \mathrm{M})$. Vitecek et al. (2008) showed that by using two traps (one for gas phase and one for liquid phase) it is possible to measure $\mathrm{NO}$ both is gas and liquid phases. The main problem of this method is the interference of internal nitrite which concentrations are much higher than NO.

Electron-spin resonance (ESR) is a well-accepted method to detect $\mathrm{NO}$ in a liquid phase. This method is based on detection of unpaired electrons that exhibit resonance in opposite orientations. This method is very specific for NO detection and its limit is in the picomolar range (Weaver et al., 2005). But the ESR spectrometer is expensive and special expertise is needed for operation. It cannot measure the emitted NO but it rather detects the trapped NO, and the online measurement of NO is not possible by this method.

Another biochemical assay to measure $\mathrm{NO}$ is the oxyhemoglobin assay. It is based on the reaction of oxyhemoglobin $\left(\mathrm{HbO}_{2}\right)$ with $\mathrm{NO}$ resulting in the production of methemoglobin (MetHb) and nitrate $\left(\mathrm{NO}_{3}^{-}\right)$. Methemoglobin is detected at $401 \mathrm{~nm}$. This method has considerable sensitivity which is in the nanomolar range (Murphy and Noack, 1994). This assay has been used by plant scientists (Cvetkovska and Vanlerberghe, 2012). Although it has a good sensitivity, the serious problem is that the reactive oxygen species oxidize $\mathrm{HbO}_{2}$ and give false positive results. The changes in $\mathrm{pH}$ can also affect the reaction. Since both ROS production and $\mathrm{pH}$ change are a part of stress response, caution should be taken while interpreting the results used via this method. NO is a free radical molecule that escapes from the site of production to target and also diffuses to the atmosphere. By the time hemoglobin assays is done there is a huge possibility that NO escapes from the sample. The techniques like oxyhemoglobin assay should be coupled with another measurement method like chemiluminescence, which can measure NO in the gas phase.

A widely used and most controversial method for NO detection is diaminofluorescein (DAF) fluorescent dyes (Foissner et al., 2000; Lamotte et al., 2004; Corpas et al., 2006; Prats et al., 2008; Cvetkovska and Vanlerberghe, 2012 and many other references). The principle of this method is based on 4,5-diaminofluorescein diacetate (DAF-2DA) diffusion into cells where the acetate groups are removed by intracellular esterases and generate 4,5diaminofluorescein; DAF-2 can also react with $\mathrm{N}_{2} \mathrm{O}_{3}$, an oxidation product of $\mathrm{NO}$, to generate the highly fluorescent DAF-2T (triazolofluorescein).

\section{THE USE OF FLUORESCENT DYES: ADVANTAGES AND CAUTIONS}

The advantage of DAF dyes that it is very easy to apply them and observe the NO fluorescence using the fluorescent or confocal microscope, which are easily available commercially and cost-effective. If NO is produced in specific sites such as guard cells, meristems or nodules or pathogeninfected cells these dyes can easily react with $\mathrm{NO}$ and give good indication about NO production. DAF dyes are relatively sensitive to NO having the detection limit in the nanomolar range and moreover no additional fluorescence is observed with $\mathrm{NO}_{2}^{-}, \mathrm{NO}_{3}^{-}, \mathrm{H}_{2} \mathrm{O}_{2}$, and $\mathrm{ONOO}^{-}$(Kojima et al., 1998).

However, the application of fluorescent dyes has been challenged by various studies. For instance, DAF2 reacts with dehydroascorbic acid (DHA) and ascorbic acid (AA) and forms fluorescent products within the similar range of fluorescence as DAF-2T (Zhang et al., 2002). Jourd'heuil (2002) was the first who suggested that DAF fluorescence is sensitive to $\mathrm{NO}$ only in the presence of superoxide $\left(\mathrm{O}_{2}^{-}\right)$or peroxynitrite $\left(\mathrm{ONOO}^{-}\right)$. 
This was confirmed in the independent study by Roychowdhury et al. (2002). The value of $\mathrm{pH}$ can also affect the fluorescence from DAF (Vitecek et al., 2008). Planchet and Kaiser (2006) compared DAF fluorescence with the chemiluminescence method and found that tobacco cell suspension that produces DAF fluorescence does not necessarily produce NO signal in chemiluminescence. Rümer et al. (2012) have shown that the cyptogein induced tobacco cells produce multiple products that generate DAF-fluorescence in vitro which is not attributed to DAF-2T, some of them are attributed to the reaction of apoplastic peroxidase, DAF and $\mathrm{H}_{2} \mathrm{O}_{2}$ in which DAF was a substrate for peroxidase. Horseradish-peroxidase plus $\mathrm{H}_{2} \mathrm{O}_{2}$ also generated DAF-fluorescence in vitro.

Carboxy-PTIO (cPTIO) is a widely used NO scavenging compound to check whether NO is responsible for the observed fluorescence. The mechanism of cPTIO effect is based on the oxidation of $\mathrm{NO}$ to $\mathrm{NO}_{2}$, and thereby it scavenges NO. But when the excess of NO is produced, $\mathrm{NO}$ reacts with $\mathrm{NO}_{2}$ and forms $\mathrm{N}_{2} \mathrm{O}_{3}$ which leads to the increased fluorescence. Therefore, the cPTIO-based NO assessment depends on NO concentrations. Moreover Rümer et al. (2012) found that the decrease in DAF fluorescence by applying CPTIO does not necessarily indicate the initial presence of $\mathrm{NO}$ since $\mathrm{CPTIO}$ can also decrease $\mathrm{H}_{2} \mathrm{O}_{2}$ production. During various stress conditions plants produce NO and ROS. Therefore, the use of DAF fluorescent dye can hamper the actual situation of NO status. On the other hand, for determing $\mathrm{NO}$ production in specific cells such as root tips or stomatal guard cells, fluorescence indicators are necessary to distinguish NO producing cells from non-producing cells. Another frequently used dye is DAF-FMDA having higher sensitivity than DAF-2DA (3 nM). But the disadvantage of DAF-FMDA is that its fluorescence depends on $\mathrm{pH}$ (Vitecek et al., 2008). Since plant cells exhibit different $\mathrm{pH}$ values at different stress conditions, the DAF-FM method should be used with caution. Another fluorescence dye is diaminorhodamine-4M (DAR-4M) (Lacza et al., 2005) but it is useful for assessment of total reactive nitrogen species (RNS) rather than for measurement of NO.

\section{CONCLUSION}

Taken together all the information presented here can teach us various things:

Fluorescent dyes are very useful for detecting NO in specific cells but, as described above, they have various disadvantages. It should be recommended to do independent measurement of DAF-2T using high-pressure liquid chromatography (Rümer et al., 2012). Always one should detect autofluorescence before adding fluorescent dyes to the samples. Only few authors used two methods for NO detection which provides necessary comparison and gives more reliable results. For instance, Planchet and Kaiser (2006) used DAF fluorescence and chemiluminescence; Bright et al. (2009) used DAF fluorescence and EPR; Cantrel et al. (2011) used DAF fluorescence and chemiluminescence assay; Gupta et al. (2013) used chemiluminescence and quantum cascade laser; DAF fluorescence and hemoglobin assay were used by Cvetkovska and Vanlerberghe (2012).

On the other hand, many studies still lacking the practice of using dual approach methods which results in low reliability because of the lack of independent verification of uncertainties generated by the restrictions of one method applied. Therefore, we recommend always using two independent methods with appropriate controls in order to obtain valuable information about $\mathrm{NO}$ concentrations and distribution in plant growth, development and stress response. In particular, we recommend using at least one gas phase method (1) and one liquid phase method (2) (See below).

\begin{tabular}{ll}
\hline Method 1 (gas phase) & Method 2 (liquid phase) \\
\hline $\begin{array}{l}\text { Chemiluminescence } \\
\text { Laser-based }\end{array}$ & NO electrodes \\
photoacoustic & $\begin{array}{l}\text { Indirect } \\
\text { chemiluminescence }\end{array}$ \\
detection & \\
Quantum cascade laser & Electron spin resonance \\
Membrane inlet mass & Oxyhemoglobin assay \\
spectrometry & Griess reagent assay \\
& Fluorescent based dyes
\end{tabular}

\section{ACKNOWLEDGMENTS}

Kapuganti J. Gupta was supported by Marie Curie Intra-European Fellowship for Career Development from EU FP 7. Abir U. Igamberdiev was supported by the Natural Sciences and Engineering
Research Council of Canada (NSERC). Kapuganti J. Gupta thanks Werner Kaiser for introducing him to NO methods.

\section{REFERENCES}

Besson-Bard, A., Griveau, S., Bedioui, F., and Wendehenne, D. (2008). Real-time electrochemical detection of extracellular nitric oxide in tobacco cells exposed to cryptogein, an elicitor of defence responses. J. Exp. Bot. 59, 3407-3414.

Bright, J., Hiscock, S. J., James, P. E., and Hancock, J. T. (2009). Pollen generates nitric oxide and nitrite: a possible link to pollen-induced allergic responses. Plant Physiol. Biochem. 47, 49-55.

Cantrel, C., Vazquez, T., Puyaubert, J., Rezé, N., Lesch, M., Kaiser, W. M., et al. (2011). Nitric oxide participates in cold-responsive phosphosphingolipid formation and gene expression in Arabidopsis thaliana. New Phytol. 189, 415-427.

Conrath, U., Amoroso, G., Kohle, H., and Sultemeyer, D. F. (2004). Non-invasive online detection of nitric oxide from plants and some other organisms by mass spectrometry. Plant J. 38, 1015-1022.

Corpas, F. J., Barroso, J. B., Carreras, A., Valderrama, R., Palma, J. M., León, A. M., et al. (2006) Constitutive arginine-dependent nitric oxide synthase activity in different organs of pea seedlings during plant development. Planta 224, 246-254.

Cvetkovska, M., and Vanlerberghe, G. C. (2012). Alternative oxidase modulates leaf mitochondrial concentrations of superoxide and nitric oxide. New Phytol. 195, 32-39.

Foissner, I., Wendehenne, D., Langebartels, C., and Durner, J. (2000). In vivo imaging of an elicitorinduced nitric oxide burst in tobacco. Plant J. 23, 817-824.

Gupta, K. J., and Kaiser, W. M. (2010). Production and scavenging of nitric oxide by barley root mitochondria. Plant Cell Physiol. 51, 576-584.

Gupta, K. J., Fernie, A. R., Kaiser, W. M., and van Dongen, J. T. (2011). On the origins of nitric oxide. Trends Plant Sci. 16, 160-168.

Gupta, K. J., Brotman, Y., Segu, S., Zeier, T., Zeier, J., Persijn, S. T., et al. (2013). The form of nitrogen nutrition affects resistance against Pseudomonas syringae pv. phaseolicola in tobacco. J. Exp. Bot. 64, 553-568.

Igamberdiev, A. U., Seregélyes, C., Manac'h, N., and Hill, R. D. (2004). NADH-dependent metabolism of nitric oxide in alfalfa root cultures expressing barley hemoglobin. Planta 219, 95-102.

Igamberdiev, A. U., Bykova, N. V., and Hill, R. D. (2006). Nitric oxide scavenging by barley hemoglobin is facilitated by a monodehydroascorbate reductase-mediated ascorbate reduction of methemoglobin. Planta 223, 1033-1040.

Jourd'heuil, D. (2002). Increased nitric oxide-dependent nitrosylation of 4, 5 diaminofluorescein by oxidants: implications for the measurement of intracellular nitric oxide. Free Radic. Biol. Med. 33, 676-684.

Kojima, H., Nakatsubo, N., Kikuchi, K., Kawahara, S., Kirino, Y., Nagoshi, H., et al. (1998). Detection and imaging of nitric oxide with novel fluorescent indicators: diaminofluoresceins. Anal. Chem. 70, 2446-2453. 
Lacza, Z., Horváth, E. M., Pankotai, E., Csordás, A., Kollai, M., Szabó, C., et al. (2005). The novel red fluorescent probe DAR-4M measures reactive nitrogen species rather than NO. J. Pharmacol. Toxicol. Methods 52, 335-340.

Lamotte, O., Gould, K., Lecourieux, D., SequeiraLegrand, A., Lebrun-Garcia, A., Durner, J., et al. (2004). Analysis of nitric oxide signaling functions in tobacco cells challenged by the elicitor cryptogein. Plant Physiol. 135, 516-529.

Leshem, Y. Y. (1996). Nitric oxide in biological systems. Plant Growth Regul. 18, 155-159.

Moeskops, B. W., Cristescu, S. M., and Harren, F. J. (2006). Sub-part-per-billion monitoring of nitric oxide by use of wavelength modulation spectroscopy in combination with a thermoelectrically cooled, continuous-wave quantum cascade laser. Opt. Lett. 31, 823-825.

Moreau, M., Lindermayr, C., Durner, J., and Klessig, D. F. (2010). NO synthesis and signaling in plantswhere do we stand? Physiol. Plant. 138, 372-383.

Mur, L. A. J., Santosa, I. E., Laarhoven, L. J. J., Holton, N. J., Harren, F. J. M., and Smith, A. R. (2005). Laser photoacoustic detection allows in planta detection of nitric oxide in tobacco following challenge with avirulent and virulent Pseudomonas syringae pathovars. Plant Physiol. 138, 1247-1258.

Mur, L. A. J., Carver, T. L. W., and Prats, E. (2006). NO way to live; the various roles of nitric oxide in plant-pathogen interactions. J. Exp. Bot. 57, 489-505.

Mur, L. A. J., Mandon, J., Cristescu, S. M., Harren, F. J., and Prats, E. (2011). Methods of nitric oxide detection in plants: a commentary. Plant Sci. 181, 509-519.

Mur, L. A. J., Mandon, J., Persijn, S., Cristescu, S., Moshkov, I., Novikova, G., et al. (2013). Nitric oxide in plants: an assessment of the current state of knowledge. AoB Plants 5:pls052. doi: 10.1093/aobpla/pls052

Murphy, M. E., and Noack, E. (1994). Nitric oxide assay using hemoglobin method. Methods Enzymol. 233, 240-250.
Planchet, E., Gupta, K. J., Sonoda, M., and Kaiser, W. M. (2005). Nitric oxide emission from tobacco leaves and cell suspensions: rate limiting factors and evidence for the involvement of mitochondrial electron transport. Plant J. 41, 732-743.

Planchet, E., and Kaiser, W. M. (2006). Nitric oxide (NO) detection by DAF fl uorescence and chemiluminescence: a comparison using abiotic and biotic NO sources. J. Exp. Bot. 57, 3043-3055.

Prats, E., Carver, T. L. W., and Mur, L. A. J. (2008). Pathogen-derived nitric oxide influences formation of the appressorium infection structure in the phytopathogenic fungus Blumeria graminis. Res. Microbiol. 159, 476-480.

Roychowdhury, S., Luthe, A., Keilhoff, G., Wolf, G., and Horn, T. F. (2002). Oxidative stress in glial cultures: detection by DAF-2 fluorescence used as a tool to measure peroxynitrite rather than nitric oxide. Glia 38, 103-114.

Rümer, S., Krischke, M., Fekete, A., Mueller, M. J., and Kaiser, W. M. (2012). DAF-fluorescence without NO: elicitor treated tobacco cells produce fluorescing DAF-derivatives not related to DAF-2 triazol. Nitric Oxide 27, 123-135.

Shah, J. K., Cochrane, D. W., De Paepe, R., and Igamberdiev, A. U. (2013). Respiratory complex I deficiency results in low nitric oxide levels, induction of hemoglobin and upregulation of fermentation pathways. Plant Physiol. Biochem. 63, 185-190.

Shibuki, K. (1990). An electrochemical microprobe for detecting nitric-oxide release in brain-tissue. Neurosci. Res. 9, 69-76.

Shirinova, A. G., Prokhorova, G. V., Ivanov, V. M., Osipova, E. A., and Chebukov, D. E. (1993). Polarographic determination of nitrite ions in grape wine. J. Anal. Chem. 48, 128-133.

Vandelle, E., and Delledonne, M. (2008). Methods for nitric oxide detection during plaintpathogen interactions. Methods Enzymol. 437, 575-594.

Vanin, A. F., Svistunenko, D. A., Mikoyan, V. D., Serezhenkov, V. A., Fryer, M. J., Baker,
N. R., et al. (2004). Endogenous superoxide production and the nitrite/nitrate ratio control the concentration of bioavailable free nitric oxide in leaves. J. Biol. Chem. 279, 24100-24107.

Vitecek, J., Reinohl, V., and Jones, R. L. (2008). Measuring NO production by plant tissues and suspension cultured cells. Mol. Plant 1, 270-284.

Weaver, J., Porasuphatana, S., Tsai, P., Budzichowski, T., and Rosen, G. M. (2005). Spin trapping nitric oxide from neuronal nitric oxide synthase: a look at several irondithiocarbamate complexes. Free Radic Res. 39, 1027-1033.

Wink, D. A., and Mitchell, J. B. (1998). Chemical biology of nitric oxide: insights into regulatory, cytotoxic, and cytoprotective mechanisms of nitric oxide. Free Radic. Biol. Med. 25, 434-456.

Zhang, X., Kim, W. S., Hatcher, N., Potgieter, K., Moroz, L. L., Gillette, R., et al. (2002). Interfering with nitric oxide measurements. 4, 5-diaminofluorescein reacts with dehydroascorbic acid and ascorbic acid. J. Biol. Chem. 277, 48472-48478.

Received: 18 January 2013; accepted: 04 March 2013; published online: 20 March 2013.

Citation: Gupta KJ and Igamberdiev AU (2013) Recommendations of using at least two different methods for measuring NO. Front. Plant Sci. 4:58. doi: $10.3389 / \mathrm{fpls} .2013 .00058$

This article was submitted to Frontiers in Plant Physiology, a specialty of Frontiers in Plant Science. Copyright (c) 2013 Gupta and Igamberdiev. This is an open-access article distributed under the terms of the Creative Commons Attribution License, which permits use, distribution and reproduction in other forums, provided the original authors and source are credited and subject to any copyright notices concerning any third-party graphics etc. 